\title{
Light-Scattering Patterns from Random Assembly of Anisotropic Rod-like Structures with their Principal Optic Axes Oriented in Cylindrical Symmetry with Respect to their Own Rod Axes*
}

\author{
Yukinobu Murakami, Norio Hayashi, ${ }^{* *}$ Takeji Hashimoto, \\ and Hiromichi KAWAI*** \\ Department of Polymer Chemistry, Faculty of Engineering, \\ Kyoto University, Kyoto, Japan.
}

(Received September 14, 1972)

\begin{abstract}
Polarized light scattering from systems having random assembly of anisotropic fibrils with finite length $L$ and infinitely thin thickness were theoretically calculated. The calculations were carried out for the fibrils randomly oriented in threedimensional space. As for orientation of optic axes within the fibrils, two types of fibrils were considered; one in which the orientation of the optic axes is fixed in a plane within the fibril and the other in which the orientation is cylindrically symmetric around the fibril axis. Differences in the scattering patterns from two types of the fibrils were discussed. By comparing the scatterings from two types of the fibrils with experimental scattering patterns, it is suggested that the fibrils which show maximum $H_{\mathrm{V}}$ scattering intensities at azimuthal angles $\mu=0^{\circ}$ and $90^{\circ}$ must be the former type of the fibrils. However for the fibrils which show maximum $H_{\mathrm{V}}$ scattering intensity at $\mu=45^{\circ}$, a unique determination of the types of the fibrils seems to be difficult by light-scattering method alone.
\end{abstract}

KEY WORDS Polarized Light Scattering / Anisotropic Fibrils / Anisotropic Rods / Cylindrically Symmetric Anisotropy /

The scattering patterns which do not exhibit the characteristics typical to spherulitic scattering ${ }^{2}$ have been occasionally observed in crystalline polymer films such as poly(tetrafluoroethylene), ${ }^{3}$ poly(chlorotrifluoroethylene), ${ }^{4}$ amylose, ${ }^{5}$ water soluble hydroxypropylcellulose,${ }^{6}$ native cellulose, ${ }^{7}$ and collagen. ${ }^{1,8}$ The scatterings called as "rodlike scattering" have been shown to arise from textures of nonspherulitic but random assembly of anisotropic fibrils.

In a previous paper, ${ }^{1}$ scattering patterns from the unoriented denatured collagen films were theoretically explained in terms of a random assembly of optically anisotropic rod-like structure.

* This work may be considered to be a supplement of the previous paper of ref 1 .

** Present address: The Central Research Laboratories, Mitsubishi Chemical Industries, Inc., HisamotoKamoi-cho, Kawasaki, Japan.

*** To whom correspondence should be sent.
Figure 1 shows the previous model used to describe the anisotropic fibrillar scattering entity oriented randomly in three-dimensional space. In the

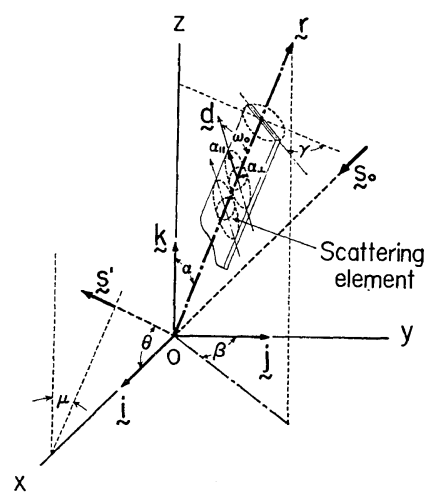

Figure 1. Model of an anisotropic rod for Model A fibrils. ${ }^{1}$ Model B fibrils have cylindrically symmetric orientation of the optic axis, i.e., randomly varying $\gamma$ within the fibrils. 
model the orientation of the optic axis which is designated as a unit vector $\mathbf{d}$ was assumed to be constant within the fibrils, so that the polar angle $\omega_{0}$ and azimuthal angle $\gamma$ were kept constant. In case of the collagen films the optic axis direction may be considered to be parallel to the tropocollagen molecules. ${ }^{9}$ The fibrils were assumed to have finite length of $L$ but infinitely thin lateral dimensions.

In this paper, we shall treat the scattering from a supplementary system to the previous model in which the fibrils have cylindrically symmetric optical anisotropy, i.e., a system in which $\gamma$ is randomly varying within the fibrils. The new model which shall be designated as "fibrils of Model B" for conveniences of further descriptions differs only in the manner of the orientation of the optic axes from the previous model ${ }^{1}$ which shall be designated as "fibrils of Model A." Differences in scattering patterns from two systems, the models $A$ and $B$, were analyzed for a better understanding of the rod-like scattering.

Such fibrils of the model B may be found when (i) the fibrils are composed of a stack of hellically twisted chain molecules parallel to the fibrillar axes with twisting periode short compared with the wavelength of visible light, when (ii) the fibrils are composed of more elementary fibrils arranged cylindrically symmetrically around their fibrillar axes, or when (iii) the fibrils themselves are twisted around their fibrillar axes with twisting period which is irregular or short compared with wavelength of the visible light like the radial fibrils of nonringed spherulites.

\section{CALCULATIONS}

As in the previous Model $\mathrm{A},{ }^{1}$ the fibrils are assumed to be composed of uniaxially anisotropic scattering elements with polarizabilities $\alpha_{\| /}$and $\alpha_{\perp}$ along and perpendicular to the optic axis, respectively. Angles $\alpha$ and $\beta$ are the orientation angles of the fibril with respect to Cartesian coordinate $O-X Y Z$ where the $O X$ and $O Z$ axes are set parallel to a unit vector $\mathbf{s}_{0}$ parallel to propagation direction of incident beam and vertical direction of the photographic system, respectively. The scattered beam parallel to a unit vector $\mathbf{s}^{\prime}$ is observed as a function of scattering angle $\theta$ and azimuthal angle $\mu$ as well as polarization conditions of ex- periments; $V_{V}$ scattering in which both polarizer and analyzer are set in the vertical direction and $\mathrm{H}_{\mathrm{V}}$ scattering in which the polarizer and analyzer are set in vertical and horizontal directions, respectively. $^{3}$

Amplitude of scattering from the fibril is given, according to Rayleigh-Gans theory, ${ }^{10}$ by

$$
E=C \int_{-L / 2}^{L / 2}(\mathbf{M} \cdot \mathbf{O}) \cos [k(\mathbf{r} \cdot \mathbf{s})] \mathrm{d} r
$$

where $\mathbf{M}$ and $\mathbf{O}$ are induced dipole moment and a unit vector parallel to the polarization direction of the analyzer set perpendicular to $O X$ axis and in between the sample and the photographic plate to record the scattering patterns. The scattering vector $\mathbf{s}$ is defined as $\mathbf{s}=\mathbf{s}_{0}-\mathbf{s}^{\prime}$ and $\mathbf{r}$ is a vector along the fibrillar axis.

For the system with random assembly of the fibrils in which interfibrillar interferences of the scattered light can be ignored, average scattered intensity is given by

$$
I=K \int_{0}^{2 \pi} \int_{0}^{\pi} E^{2} \sin \alpha \mathrm{d} \alpha \mathrm{d} \beta
$$

As before we shall assume that the fibril is homogeneous so that the effective induced dipole moment under a given polarization condition is independent of $r$. Consequently

$$
E^{2}=C^{2}(\mathbf{M} \cdot \mathbf{O})^{2}\left[\int_{-L / 2}^{L / 2} \cos [k(\mathbf{r} \cdot \mathbf{s})] \mathrm{d} r\right]^{2}
$$

The integration can be readily carried out as shown in a previous paper. ${ }^{1}$ In a more general case in which there is optical heterogeneities within the fibrils, (M.O) depends upon $r$, and the calculations become more complex, the effect of which shall be discussed elsewhere. ${ }^{11}$ It is also assumed that the fibrils are identical with respect to their size. The effect of the size distribution on the scattering shall be also discussed elsewhere. ${ }^{11}$

The effective induced dipole moment is obtained by evaluating the components of the incident field $\mathbf{E}_{0}=\left(E_{0 x}, E_{0 y}, E_{0 z}\right)$ along the principal axes of the polarizability ellipsoid of the scattering element $\left(\mathbf{Q}^{t} \mathbf{E}_{0}\right)$ and by evaluating the components of the induced dipole moment thus calculated $\left(\alpha \mathbf{Q}^{t} \mathbf{E}_{0}\right)$ along the polarization direction of the analyzer $\left(\mathbf{Q} \alpha \mathbf{Q}^{t} \mathbf{E}_{0}\right)$. Thus if matrix of an orthogonal coordinate transformation between $O-X Y Z$ system and the principal axes of the scattering element 
is given by $\mathbf{Q}$, and for vertically polarized incident beam in which $\mathbf{E}_{0}=\left(0,0, E_{0}\right)$, the induced dipole moment under $H_{\mathrm{V}}$ and $V_{\mathrm{V}}$ polarizations are given by

$$
\begin{aligned}
& (\mathbf{M} \cdot \mathbf{O})_{H_{\mathrm{v}}}=\mathbf{Q} \boldsymbol{\alpha} \mathbf{Q}^{t}\left(\begin{array}{c}
0 \\
0 \\
E_{0}
\end{array}\right) \cdot \mathbf{j} \\
& (\mathbf{M} \cdot \mathbf{O})_{V_{\mathrm{v}}}=\mathbf{Q} \boldsymbol{\alpha} \mathbf{Q}^{t}\left(\begin{array}{c}
0 \\
0 \\
E_{0}
\end{array}\right) \cdot \mathbf{k}
\end{aligned}
$$

where $\mathbf{j}$ and $\mathbf{k}$ are the unit vectors parallel to $O Y$ and $O Z$ axes, and $\alpha$ is a polarizability tensor along the principal axes given by

$$
\boldsymbol{\alpha}=\left(\begin{array}{lll}
b_{\mathrm{t}} & 0 & 0 \\
0 & b_{\mathrm{t}} & 0 \\
0 & 0 & b_{\mathrm{r}}
\end{array}\right)
$$

$\mathbf{Q}^{t}$ is a transposed matrix of $\mathbf{Q}$. $b_{\mathrm{t}}$ and $b_{\mathrm{r}}$ are defined as $b_{\mathrm{t}}=\alpha_{\perp}-\alpha_{\mathrm{s}}$, and $b_{\mathrm{r}}=\alpha_{\| /}-\alpha_{\mathrm{s}}$ where $\alpha_{\mathrm{s}}$ is polarizability of medium surrounding the fibril as in a previous paper. ${ }^{1}$

The transformation matrix $\mathbf{Q}$ is obviously given by a product of orthogonal matrices of coordinate transformations.

$$
\mathbf{Q}=\mathbf{Q}_{1} \mathbf{Q}_{2}
$$

where $\mathbf{Q}_{1}$ and $\mathbf{Q}_{2}$ are given by

$\mathbf{Q}_{1}=\left(\begin{array}{lll}\cos \alpha \sin \beta, & -\cos \beta, & \sin \alpha \sin \beta \\ \cos \alpha \cos \beta, & \sin \beta, & \sin \alpha \cos \beta \\ -\sin \alpha, & 0, & \cos \alpha\end{array}\right)$
$\mathbf{Q}_{2}=\left(\begin{array}{lll}\cos \omega_{0} \cos \gamma, & -\sin \gamma, & \sin \omega_{0} \cos \gamma \\ \cos \omega_{0} \sin \gamma, & \cos \gamma, & \sin \omega_{0} \sin \gamma \\ -\sin \omega_{0}, & 0, & \cos \omega_{0}\end{array}\right)$

Now from eq 4 to 7 , it follows that

$$
\begin{aligned}
(\mathbf{M} \cdot \mathbf{O})_{\mathrm{H}_{\mathrm{v}}} & \\
= & \left(b_{\mathrm{r}}-b_{\mathrm{t}}\right) E_{0}\left[\left(\cos ^{2} \omega_{0}\right.\right. \\
& \left.-\sin ^{2} \omega_{0} \cos ^{2} \gamma\right) \sin \alpha \cos \alpha \cos \beta \\
& +\sin \omega_{0} \cos \omega_{0} \cos \gamma\left(\cos ^{2} \alpha-\sin ^{2} \alpha\right) \cos \beta \\
& -\sin ^{2} \omega_{0} \sin \gamma \cos \delta \sin \alpha \sin \beta \\
& \left.+\sin \omega_{0} \cos \omega_{0} \sin \gamma \cos \alpha \sin \beta\right] \\
(\mathbf{M} \cdot \mathbf{O})_{\mathrm{V}_{\mathrm{v}}} & \\
= & \left(b_{\mathrm{r}}-b_{\mathrm{t}}\right) E_{0}\left[\left(\cos ^{2} \omega_{0}-\sin ^{2} \omega_{0} \cos ^{2} \gamma\right) \cos ^{2} \alpha\right. \\
& +\sin ^{2} \omega_{0} \cos ^{2} \gamma \\
& \left.-2 \sin \omega_{0} \cos \omega_{0} \cos \gamma \sin \alpha \cos \alpha\right]+b_{\mathrm{t}} E_{0}
\end{aligned}
$$

The effect of the orientations of optic axes on the light scattering can be seen in eq 8 and 9 .

For the fibrils of the Model B in which $\gamma$ is varying randomly with constant angle $\omega_{0}$, only an average of the effective induced dipole moment with respect to $\gamma$ contributes to the scattering, so that it follows that

$$
\begin{aligned}
& \left\langle(\mathbf{M} \cdot \mathbf{O})_{H_{\mathrm{v}}}\right\rangle_{\mathrm{av}} \\
& \quad=\left(b_{\mathrm{r}}-b_{\mathrm{t}}\right) E_{0}\left[\frac{3 \cos ^{2} \omega_{0}-1}{2}\right] \sin \alpha \cos \alpha \cos \beta
\end{aligned}
$$

and

$$
\begin{aligned}
\left\langle(\mathbf{M} \cdot \mathbf{O})_{V_{\mathrm{v}}}\right\rangle_{\mathrm{av}} & \\
= & \left(b_{\mathrm{r}}-b_{t}\right) E_{0}\left\{\left[\frac{3 \cos ^{2} \omega_{0}-1}{2}\right] \cos ^{2} \alpha\right. \\
& \left.+\frac{1}{2} \sin ^{2} \omega_{0}\right\}+b_{\mathrm{t}} E_{0}
\end{aligned}
$$

Consequently the average values of $\left\langle(\mathbf{M} \cdot \mathbf{O})_{H_{\mathrm{V}}}\right\rangle_{\mathrm{av}}^{2}$ and $\left\langle(\mathbf{M} \cdot \mathbf{O})_{V_{v}}\right\rangle_{\text {av }}^{2}$ should be used for $(\mathbf{M} \cdot \mathbf{O})^{2}$ in eq 2 and 3 in order to calculate the $H_{\mathrm{V}}$ aud $V_{\mathrm{V}}$ scatterings from the fibrils, respectively. From eq 10 it is expected that the value of $\omega_{0}$ affects only the absolute intensity of $\boldsymbol{H}_{\mathrm{V}}$ scattering but not the relative intensity distributions, while from eq 11 both the absolute intensity and the relative intensity distributions are affected by the value of $\omega_{0}$ for the $V_{\mathrm{V}}$ scattering.

On the other hand, for the fibrils of the Model A, the orientation of optic axes is fixed to a particular value of $\gamma$ within the fibrils, but the fibrils as a whole are randomly rotated around their axes to result in randomly varying $\gamma$ in macroscopic scale. In this case the intensity of scattering from the fibril depends upon $\left\langle(\mathbf{M} \cdot \mathbf{O})_{H_{\mathrm{v}}}^{2}\right\rangle_{\mathrm{av}}$ and $\left\langle(\mathbf{M} \cdot \mathbf{O})_{V_{\mathrm{v}}}^{2}\right\rangle_{\mathrm{av}}$. Thus average $H_{\mathrm{V}}$ and $V_{\mathrm{V}}$ intensities over all volumes of $\gamma$ for the fibril oriented in a given direction specified $\alpha$ and $\beta$ in space are given by substituting $\left\langle(\mathbf{M} \cdot \mathbf{O})_{H_{\mathrm{V}}}^{2}\right\rangle_{\mathrm{av}}$ and $\left\langle(\mathbf{M} \cdot \mathbf{O})_{V_{\mathrm{v}}}^{2}\right\rangle_{\mathrm{av}}$ into $(\mathbf{M} \cdot \mathbf{O})^{2}$ in eq 3. From eq 8 and 9 , it follows that

$$
\begin{aligned}
\left\langle(\mathbf{M} \cdot \mathbf{O})_{H_{\mathrm{v}}}^{2}\right\rangle_{\mathrm{av}} \\
=\left(b_{\mathrm{r}}-b_{\mathrm{t}}\right)^{2} E_{0}^{2}\left\{\left[\cos ^{4} \omega_{0}-\cos ^{2} \omega_{0} \sin ^{2} \omega_{0}\right.\right. \\
\left.\quad+\left(\frac{3}{8}\right) \sin ^{4} \omega_{0}\right] \sin ^{2} \alpha \cos ^{2} \alpha \cos ^{2} \beta \\
\quad+\left(\frac{1}{2}\right) \sin ^{2} \omega_{0} \cos ^{2} \omega_{0} \cos ^{2} 2 \alpha \cos ^{2} \beta \\
\quad+\left(\frac{1}{8}\right) \sin ^{4} \omega_{0} \sin ^{2} \alpha \sin ^{2} \beta \\
\left.\quad+\left(\frac{1}{2}\right) \sin ^{2} \omega_{0} \cos ^{2} \omega_{0} \cos ^{2} \alpha \sin ^{2} \beta\right\}
\end{aligned}
$$


Light Scattering from Random Assembly of Anisotropic Rods

$$
\begin{aligned}
\left\langle(\mathbf{M} \cdot \mathbf{O})_{V_{\nabla}}^{2}\right\rangle_{\mathrm{av}} \\
=\left(b_{\mathrm{r}}-b_{\mathrm{t}}\right)^{2} E_{0}{ }^{2}\left\{\left[\cos ^{4} \omega_{0}-\sin ^{2} \omega_{0} \cos ^{2} \omega_{0}\right.\right. \\
\left.\quad+\left(\frac{3}{8}\right) \sin ^{4} \omega_{0}\right] \cos ^{4} \alpha+\left(\frac{3}{8}\right) \sin ^{4} \omega_{0} \\
\quad+2 \sin ^{2} \omega_{0} \cos ^{2} \omega_{0} \sin ^{2} \alpha \cos ^{2} \alpha \\
\left.\quad+\left[\cos ^{2} \omega_{0}-\left(\frac{3}{4}\right) \sin ^{2} \omega_{0}\right] \cos ^{2} \alpha \sin ^{2} \omega_{0}\right\} \\
\quad+2\left(b_{\mathrm{r}}-b_{\mathrm{t}}\right) b_{\mathrm{t}} E_{0}{ }^{2}\left[\left(\frac{3 \cos ^{2} \omega_{0}-1}{2}\right) \cos ^{2} \alpha\right. \\
\left.\quad+\left(\frac{1}{2}\right) \sin ^{2} \omega_{0}\right]+b_{\mathrm{t}}{ }^{2} E_{0}{ }^{2}
\end{aligned}
$$

Consequently, in contrast to the scattering from the Model B fibrils both the absolute intensities and the relative intensity distributions of $H_{\mathrm{V}}$ and $V_{\mathrm{V}}$ scatterings depend upon the angle $\omega_{0}$ as previously reported for the fibrils of the Model A. ${ }^{1}$

From eq 2 to 7,10 , and $11, H_{\mathrm{V}}$ and $V_{\mathrm{V}}$ scattered intensities for the fibrils of the Model $B$ can be calculated.

$$
\begin{aligned}
I_{H_{\mathrm{V}}}= & (1 / 16) K^{2} \pi^{2} L^{2}\left(b_{\mathrm{r}}-b_{\mathrm{t}}\right)^{2}\left[P_{2}\left(\cos \omega_{0}\right)\right]^{2} \\
& \times\left[(35 A-30 B+3 C) \cos ^{4}(\theta / 2) \sin ^{2} \mu \cos ^{2} \mu\right. \\
& \left.-(5 A-6 B+C) \cos ^{2}(\theta / 2)+(A-2 B+C)\right](14) \\
I_{V_{\mathrm{v}}}= & (1 / 16) K^{2} \pi^{2} L^{2}\left(b_{\mathrm{r}}-b_{\mathrm{t}}\right)^{2}\left\{\left[P_{2}\left(\cos \omega_{0}\right)\right]^{2}\right. \\
& \times\left[(35 A-30 B+3 C) \cos ^{4}(\theta / 2) \cos ^{4} \mu\right. \\
& -6(5 A-6 B+C) \cos ^{2}(\theta / 2) \cos ^{2} \mu \\
& +3(A-2 B+C)]+8 P_{2}\left(\cos \omega_{0}\right)\left[\left(\frac{1}{2}\right) \sin ^{2} \omega_{0}+p\right] \\
& \times\left[(3 B-C) \cos ^{2}(\theta / 2) \cos ^{2} \mu-(B-C)\right] \\
& \left.+8\left[\left(\frac{1}{2}\right) \sin ^{2} \omega_{0}+p\right]^{2} C\right\}
\end{aligned}
$$

where the quantities, $p, A, B$, and $C$ are defined in a similar way as in a previous paper. ${ }^{1}$

$$
\begin{aligned}
& p=\left(\alpha_{\perp}-\alpha_{\mathrm{s}}\right) /\left(\alpha_{\| /}-\alpha_{\perp}\right) \\
& A=\frac{1}{3 U^{2}}-\left(\frac{2 U^{2}-1}{4 U^{5}}\right) \sin 2 U-\frac{1}{2 U^{4}} \cos (2 U) \\
& B=\frac{1}{U^{2}}-\frac{1}{2 U^{3}} \sin 2 U \\
& C=\frac{2}{U} S_{\mathrm{i}}(2 U)-\frac{1}{U^{2}}+\frac{1}{U^{2}} \cos (2 U) \\
& U=\left(2 \pi / \lambda^{\prime}\right) L \sin (\theta / 2)
\end{aligned}
$$

$\boldsymbol{P}_{2}(X)$ is the second order Legendre function defined by $P_{2}(X)=\left(3 X^{2}-1\right) / 2$, and $S_{\mathrm{i}}(U)$ is the sine function defined by

$$
S_{\mathrm{i}}(U)=\int_{0}^{U}(\sin X) / X \mathrm{~d} X .
$$

$\lambda^{\prime}$ is wavelength of light in the medium, and the constant $K$ related to the absolute intensity is identical to that in a previous paper.

As expected from eq 14, the intensity distributions of the $H_{\mathrm{V}}$ scattering with respect to $\theta$ depend only upon length $L$ of the fibrils and have a four fold symmetry with respect to the azimuthal angle $\mu$. The $H_{\mathrm{V}}$ scattering depends only upon $\boldsymbol{P}_{2}$ $\left(\cos \omega_{0}\right)$ in contrast to that of the previous model, Model A which depends also upon $P_{4}\left(\cos \omega_{0}\right)$, the fourth order Legendre function as discussed in eq 6 of ref 1 . Moreover, the angle $\omega_{0}$ affects only the absolute intensity, which is very much in contrast to the scattering from the fibrils of the Model $\mathrm{A}$ in which $\omega_{0}$ affects also the angular distributions with respect to $\mu$, as discussed in detail in eq 6 of ref 1 .

On the other hand, $V_{V}$ scattering intensity distributions depend upon the angle $\omega_{0}$ as well as the length $L$ as seen in eq 15. As in the previous case, $V_{\mathrm{V}}$ scattering is composed of the terms with circular symmetry, two-fold and four-fold symmetries with respect to $\mu$. A difference of $V_{\mathrm{V}}$ scatterings from two types of fibrils, the Model $\mathrm{A}$ and the Model B, may be seen by comparing eq 15 with eq 7 of ref 1 . The scattering from the fibrils of the Model B depends only upon $\boldsymbol{P}_{2}$ term, while that from the fibrils of the Model A depends upon $\boldsymbol{P}_{4}$ term as well.

The length of the fibrils of the Model B can be estimated by utilizing $H_{\mathrm{V}}$ scattering intensity distributions with respect to $\theta$ at $\mu=0^{\circ}$ and $\mu=45^{\circ}$ (eq 14),

$$
\begin{aligned}
& \left(I_{\mathrm{H}_{\mathrm{v}}}\right)_{\mu=45^{\circ}}-\left(I_{\mathrm{H}_{\mathrm{v}}}\right)_{\mu=0^{\circ}} \\
& \quad=K_{3}\left[\boldsymbol{P}_{2}\left(\cos \omega_{0}\right)\right]^{2}(35 A-30 B+3 C)
\end{aligned}
$$

From eq 21 which is valid for small $\theta$, the length of the fibrils is determined by using the relationship

$$
U_{\max }=4.80=\left(2 \pi L / \lambda^{\prime}\right) \sin \left(\theta_{\max } / 2\right)
$$

The relationship is exactly identical to that for the Model A. ${ }^{1}$

\section{NUMERICAL CALCULATIONS}

The results of numerical calculations are shown in Figures 2, 4, and 5 for Model B. The calculations were carried out for a value of $L$ specified by $L / \lambda^{\prime}=40$ as in the previous calculations for the Model A. 
Figure 2 shows the result of $H_{\mathrm{V}}$ scattering for a particular value of $\omega_{0}$ for which $\boldsymbol{P}_{2}\left(\cos \omega_{0}\right)$ becomes unity. The angular dependences of the $H_{\nabla}$ pattern are independent of $\omega_{0}$. The absolute intensity of the pattern is maximum for $\omega_{0}=0^{\circ}$ and becomes zero at $\omega_{0}$ satisfying $P_{2}\left(\cos \omega_{0}\right)=0$ as which the fibrils become optically isotropic.

The effect of $\omega_{0}$ on the $H_{\mathrm{V}}$ patterns is markedly different from that on the $H_{\nabla}$ patterns for the Model A as shown in Figure 3 (cited from Figure 9 of ref 1) in which the $H_{\mathrm{V}}$ patterns were shown

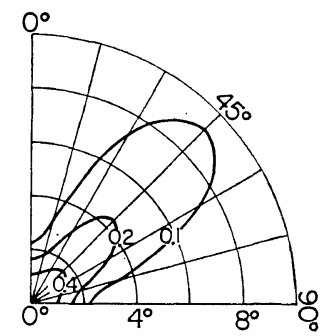

Figure 2. $H_{\mathrm{V}}$ scattering pattern from the fibrils of Model B. to exhibit $\times$-type scattering with maximum intensities at odd multiples of $\mu=45^{\circ}$ for $\omega_{0}$ from $0^{\circ}$ to $30^{\circ} 33^{\prime}$, (+)-type scattering with maximum intensities at $\mu= \pm 0^{\circ}$ and $\pm 90^{\circ}$ for $\omega_{0}$ from $30^{\circ} 33^{\prime}$ to $70^{\circ} 07^{\prime}$ and the $\times$-type scattering for $\omega_{0}$ from $70^{\circ} 07^{\prime}$ to $90^{\circ}$.

In contrast to the $H_{\mathrm{V}}$ scattering, the angular dependences of the $V_{\mathrm{V}}$ scattering depend upon $\omega_{0}$ even for Model B as shown in Figures 4 and 5 for the value of $p=-0.27$ and 0.0 , respectively, in which the patterns for the Model A and B are drawn by broken and solid lines, respectively.

The patterns drawn by the broken lines are identical to those shown in Figures 10 and 11 in a previous paper. ${ }^{1}$ The differences of $V_{\mathrm{V}}$ patterns for Model A and Model B are due to the differences in the relative contribution of orientation and density fluctuations, so that the differences become smaller for values of $p$ for which the density contributions become larger.

Values listed in brackets $(a, b)$ beneath each scattering patterns are those indicating relative

\section{Hv patterns $\left(L / \lambda^{\prime}=40\right)$}
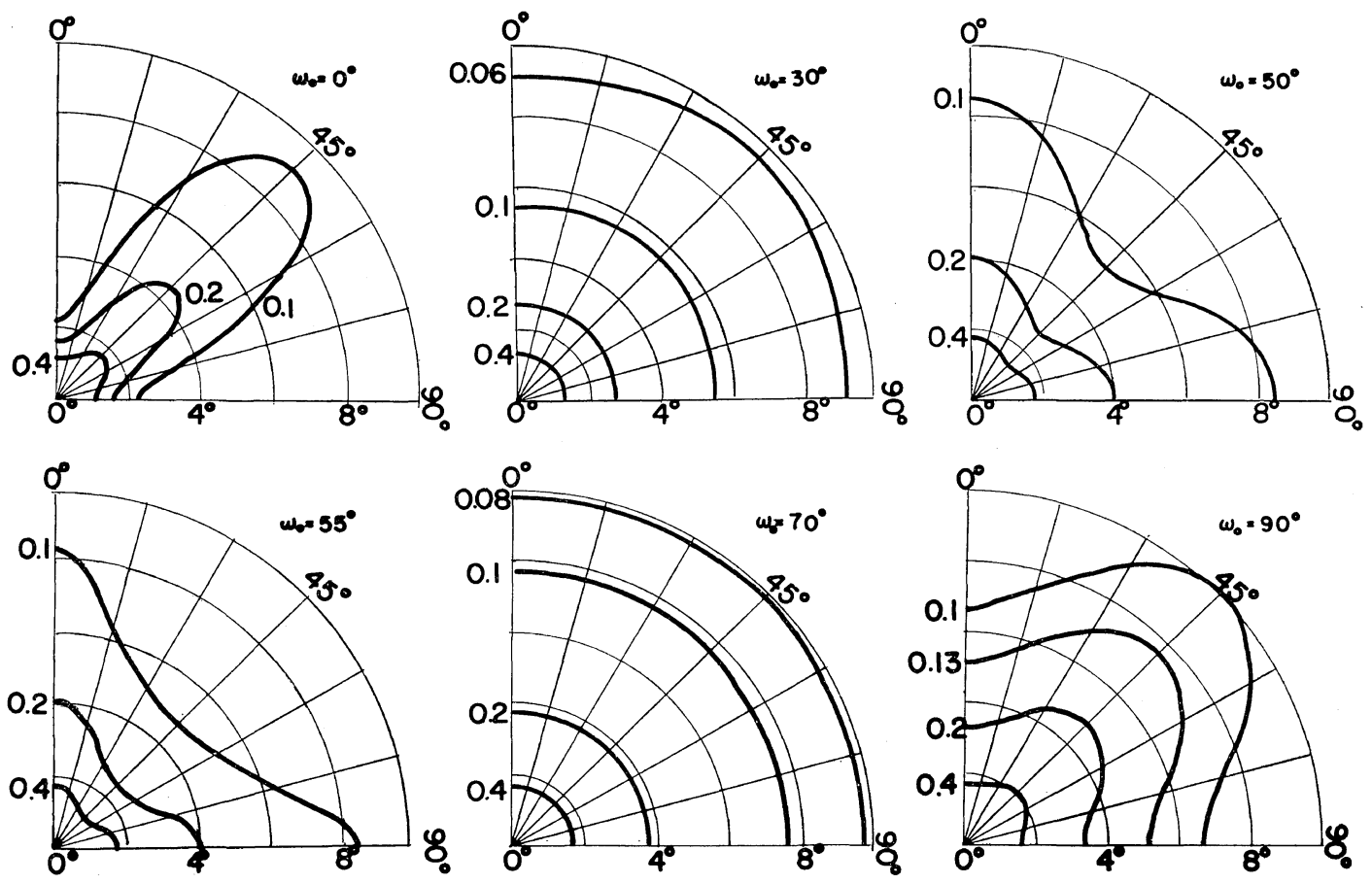

Figure 3. $H_{\mathrm{V}}$ scattering patterns from the fibrils of Model A for various values of $\omega_{0}$. The patterns are identical to those of Figure 9 of ref 1. 
Light Scattering from Random Assembly of Anisotropic Rods

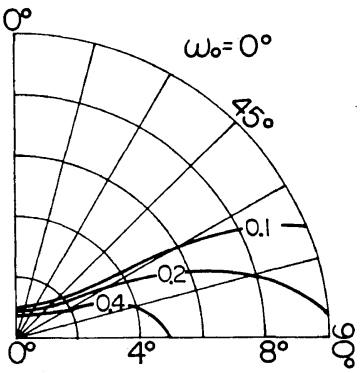

$(1.000,1.000)$

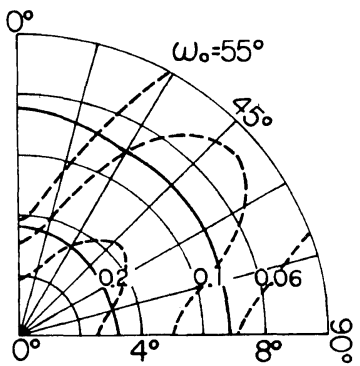

$(1.000,0.048)$

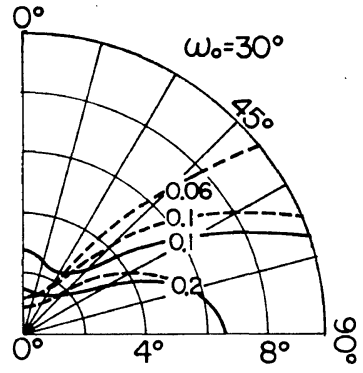

$(1.000,0.420)$

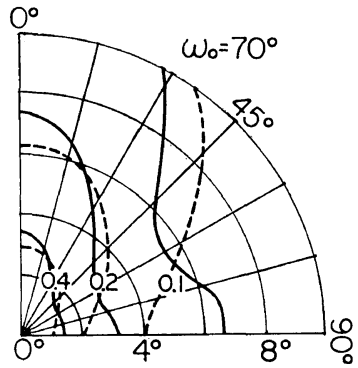

$(1.000,0.148)$

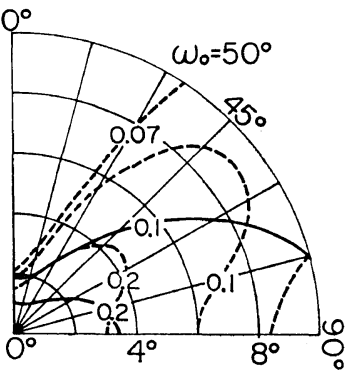

$(1.000 .0 .061)$

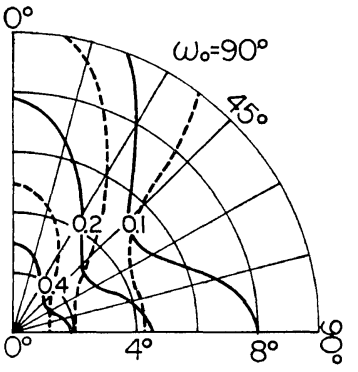

$(1.000,0.285)$

Figure 4. $V_{\mathrm{V}}$ scattering patterns from the fibrils of Model A (broken lines) and Model $\mathbf{B}$ (solid lines) for $p=-0.27$ and for various values of $\omega_{0}$.

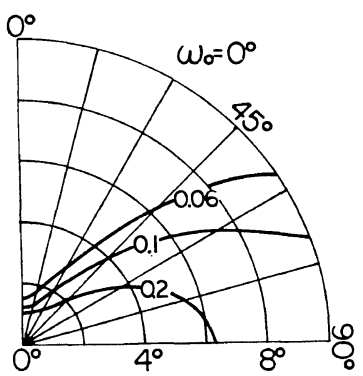

$(0.942,2.143)$

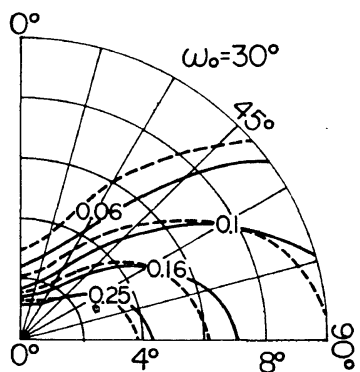

(0.942, 1.563)

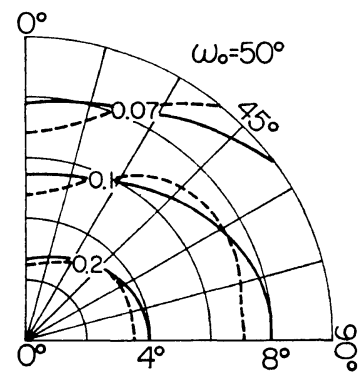

(0.942, 1.204)

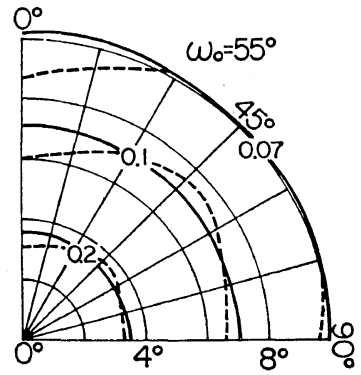

(0.942.1.191)

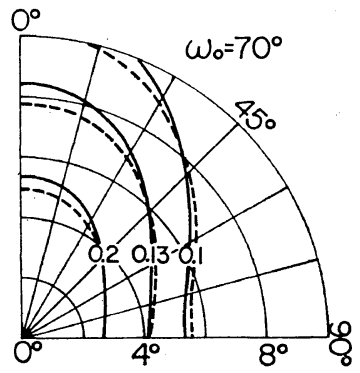

(0.942, I.291)

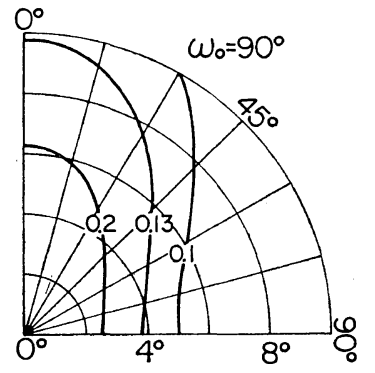

(0.942, 1.429)

Figure 5. $\quad V_{\mathrm{V}}$ scattering patterns from the fibrils of Model A (broken lines) and Model B (solid lines) for $p=0$ and for various values of $\omega_{0}$. 


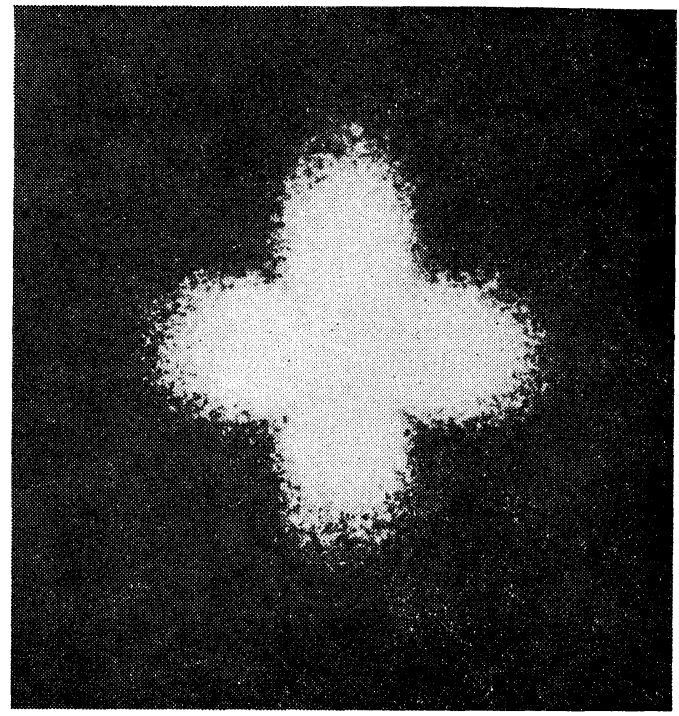

Figure 6. $H_{\mathrm{V}}$ scattering patterns from $\mathrm{RC}-1$ collagen films.

intensity changes upon changing the values of $\omega_{0}$ for models, $\mathrm{A}$ and $\mathrm{B}$, respectively when intensity levels of the $V_{\mathrm{V}}$ pattern for $\omega_{0}=0^{\circ}$ and $p=-0.27$ are chosen as unity.

In Figure 6, $H_{\mathrm{V}}$ scattering pattern is shown for the denatured collagen films previously studied. ${ }^{1}$ By comparing Figures 2 and 3 with Figure 6, the +-type scattering must be interpreted as arising from the fibrils of Model A rather than those of Model B. Consequently, for any fibrils which give the $(+)$-type scatterings the optic axes must be essentially oriented in a special plane of the fibrils, keeping $\gamma$ constant within the fibrils and $\omega_{0}$ constant between $30^{\circ} 33^{\prime}$ and $70^{\circ} 07^{\prime}$.

Thus in case of the collagen films, the tropocollagen molecules, the chain axes of which are parallel to the optic axes may be considered to be stacked side-by-side with a polar angle $\omega_{0}$ in the range of about $30^{\circ} 33^{\prime}$ to $70^{\circ} 07^{\prime}$ with respect to the fibrilar axes as in the fibrils of Model A rather than being stacked cylindrically symmetrically around the fibrilar axes as in the fibrils of Model B.

In Figure 7, the $\times$-type $H_{\mathrm{V}}$ scattering is shown for poly(tetrafluoroethylene) films. The films were made by heat treating the dispersion for $2 \mathrm{hr}$ at $412^{\circ} \mathrm{C}$ and subsequent natural cooling to room temperature.

The $x$-type scattering pattern may arise either

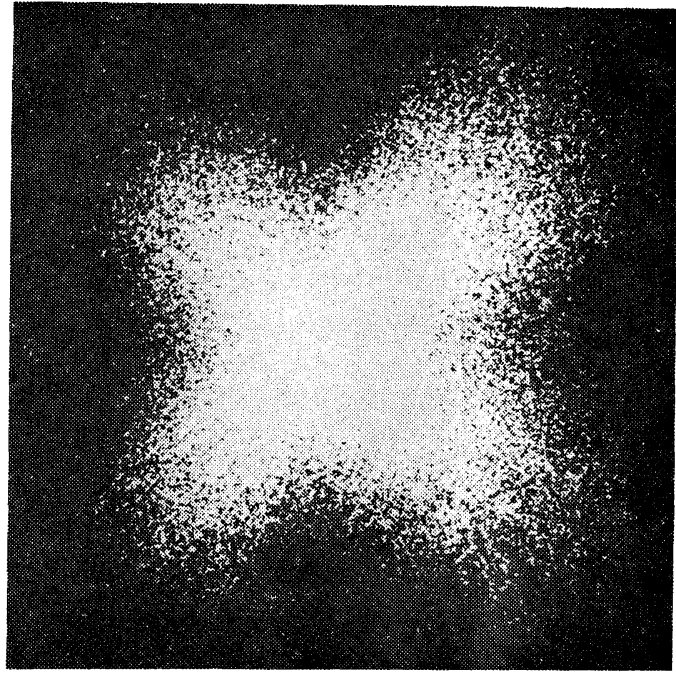

Figure 7. $H_{\mathrm{V}}$ scattering patterns from the poly(tetrafluorethylene) films.

from the fibrils of Model A with the polar angle $\omega_{0}$ in the range characteristic to the scattering or from the fibrils of Model B, so that the problem arises as to the proper choice of models. Although there are small differences in the angular dependences of $H_{\mathrm{V}}$ and $V_{\mathrm{V}}$ scattering from the fibrils of Models $\mathrm{A}$ and $\mathrm{B}$, the differences are modified by other factors such as sized distribution, internal heterogeneities and finite lateral dimensions of the fibrils. ${ }^{12,13}$ Consequently, in this case it is not feasible to determine uniquely the model from light scattering experiment alone. The determination of the model from $V_{\mathrm{V}}$ scattering experiments requires a knowledge of $\alpha_{\mathrm{s}}$ which is usually difficult to obtain.

\section{CONCLUSIONS}

$H_{\mathrm{V}}$ and $V_{\mathrm{V}}$ light scatterings from systems with two types of anisotropic fibrils are compared. In fibrils of type $A$, the principal optic axis is oriented in a special plane of the fibril indicated by azimuthal angle $\gamma$ and with constant polar angle $\omega_{0}$ (Figure 1), while in fibrils of type $\mathbf{B}$, the optic axis orients cylindrically symmetrically around the fibril axis. By comparing the calculated results for the models with experimental patterns, it is shown that the previously observed (+)-type $\mathrm{H}_{\mathrm{V}}$ scatterings from the collagen films and from other crystalline polymer films must be scatterings 
from type A fibrils. This may indicate, in case of the collagen films, that the tropocollagen molecules constituting the fibrils are packed parallel to the others with polar angle $\omega_{0}$ in a range about $30^{\circ} 33^{\prime}$ to $70^{\circ} 07^{\prime}$ in a special plane of the fibrils. However for the fibrils which give the $x$-type $\mathrm{H}_{\mathrm{V}}$ scattering patterns it is difficult to select a proper model by light-scattering experiment alone.

\section{REFERENCES}

1. M. Moritani, N. Hayashi, A. Utsuo, and H. Kawai, Polymer J., 2, 74 (1971).

2. R. S. Stein and M. B. Rhodes, J. Appl. Phys., 31, 1873 (1960).

3. M. B. Rhodes and R. S. Stein, ibid., 39, 4903 (1968).

4. N. Hayashi, Y. Murakami, M. Moritani, T.
Hashimoto and H. Kawai, Polymer $J$. in press. 5. J. Borch, R. Muggli, A. Sarko, and R. H. Marchessault, J. Appl. Phys., 42, 4570 (1971).

6. R. J. Samuels, J. Polym. Sci., Part A-2, 7, 1197 (1969),

7. J. Borch and R. H. Marchessault, ibid., Part C, 28, 153 (1969).

8. J.C.W. Chien and E.P. Chang, to be published.

9. A. Rich and F. H. C. Crick, J. Mol. Biol., 3, 483 (1961).

10. H. C. van de Hulst, "Light Scattering by Small Particles", John Wiley and Sons, Inc., New York, N. Y., 1957.

11. T. Hashimoto, N. Hayashi, Y. Murakami and H. Kawai, to be submitted to Polymer J.

12. N. Hayashi and H. Kawai, ibid., 3, 140 (1972).

13. M. Matsuo, S. Nomura, T. Hashimoto and $\mathrm{H}$. Kawai, to be submitted to Polymer $J$. 